\title{
La categoría de normalidad filosófica de Francisco Romero reguladora de la historia de la filosofía en Latinoamérica y Colombia
}

\section{Francisco Romero's “philosophical normality" category, as a regulator of Latin American and Columbian history}

\author{
Pedro Pablo Rivas Osorio \\ Doctor en Filosofía de la Universidad Nacional Autónoma de México \\ Director Centro de Estudios e Investigaciones Latinoamericanas - CEILAT- Universidad de Nariño. Docente tiempo \\ completo Universidad de Nariño. \\ Director del grupo de Investigación Ágora Latinoamericana - CEILAT- Universidad de Nariño. \\ Email: pasto2314@gmail.com
}

Mayerli Alejandra Deraso Andrade

Licenciada en Filosofía y Letras de la Universidad de Nariño,

Maestranda en Estudios Latinoamericanos del Centro de Estudios e Investigaciones

Latinoamericanas - CEILAT- Universidad de Nariño.

Docente hora cátedra de la Universidad de Nariño.

Miembro del grupo de Investigación Ágora Latinoamericana.

Email: mayerlideraso@hotmail.com

Doi: https://doi.org/10.22267/rceilat.204647.87

\section{Resumen}

Este texto tiene como propósito tratar sobre las categorías de "Normalidad Filosófica” y "Fundadores” de Francisco Romero y las contribuciones hechas al respecto por algunos pensadores latinoamericanos y colombianos. Considerando el trabajo de Romero, la filosofía en Latinoamérica a inicios del siglo XX se caracterizó por ser más rigurosa y sistemática, cuyos rasgos son consecuencia de la etapa de la normalidad filosófica, que mediante técnicas y métodos académicos configuró a la filosofía como modelo rector para la historia de la filosofía en Latinoamérica. Sin embargo, han tenido lugar dos discusiones relevantes que son citadas en este trabajo como afirmación y negación de la "Normalidad Filosófica" y "Fundadores", con el fin de demostrar que algunos pensadores consideran y reconocen estos conceptos como una idea de proyecto filosófico, que ha conducido a la toma de conciencia de hacer filosofía latinoamericana desde la concepción occidental. Por otro lado, otros pensadores se alejan de la opinión 
anterior al considerar que es una forma de delimitar la historia y un requisito impuesto al modo de hacer filosofía, profesionalizando y limitando el quehacer de esta actividad. Estas afirmaciones se identifican como dos posibilidades para asentar y repensar la historia de las ideas filosóficas.

Palabras clave: Normalidad Filosófica; Fundadores; Filosofía en Latinoamérica; Filosofía en Colombia; Historia de la Filosofía en Latinoamérica.

\begin{abstract}
The purpose of this paper is to talk about the terms "Philosophic Normality" and "Founders" by Francisco Romero, and the contributions made on the subject by some Latin-American and Colombian thinkers. Latin American philosophy at the beginning of XX century was characterized for being rigorous and systematic according to Romero. The characteristics of Latin American philosophy were a consequence of such stage of "philosophic normality", which, by means of academic methods and techniques, set general philosophy as a reference model for the history of philosophy in Latin America.

However, two relevant discussions have taken place, both cited in this paper, as affirmation and negation of "Philosophic Normality" and "Founders", in order to demonstrate that some thinkers acknowledge these concepts as an idea of a philosophic project, that has led to awareness of a Latin-American philosophy from an eastern perspective. On the other hand, other thinkers set themselves away from the previous point of view, considering it a way of narrowing history and an imposed requirement to the way of creating philosophy, by means of professionalizing and limiting the endeavor of this activity. These claims are identified as two possibilities to settle and rethink the history of philosophic ideas.
\end{abstract}

Key words: Philosophic Normality; Founders; Philosophy in Latin America; Philosophy in Colombia; History of Philosophy in Latin America.

\section{Introducción}

Normalidad filosófica es una categoría debida al filósofo español-argentino Francisco Romero (1891-1962). Por una parte, indica una fase de la historia de la filosofía en Latinoamérica; por otra, busca organizar y definir la filosofía desde lo institucional como ciencia de estudio que se hace a partir de técnicas y normas académicas orientadas en el pensamiento occidental europeo.
Según Dussel (1970), Francisco Romero llega a la Argentina aun siendo niño y se forma en la carrera militar en el Colegio Militar de Buenos Aires, fue autodidacta en el estudio de la filosofía y discípulo de Alejando Korn (1860-1936); profesor de la Facultad de Filosofía en Buenos Aires y de la Facultad de Humanidades de la Universidad de La Plata; Korn convenció a Romero de abandonar la carrera militar para que lo remplazara como pro- 
fesor. Algunas contribuciones hechas por Romero a la academia: la fundación de la Biblioteca Losada (1939), de la Revista Realidad (1947) y el proyecto de continuidad de la Biblioteca de Occidente de Ortega y Gasset. Se dice que Romero tuvo "cuatro conductos que le permitían expresar su pensar filosófico: la amistad siempre abierta al diálogo personal con colegas y alumnos; la cátedra de Buenos Aires y La Plata; la sociedad kantiana y los artículos "informativos" en diversas revistas” (Dussel, 1970, p.83).

Para Romero (1952) fue de gran importancia la presencia del filósofo español Ortega y Gasset (1883-1955) en Buenos Aires en los años 19161917, y las contribuciones de sus clases públicas sobre la introducción de su pensamiento, porque representó para su país una preparación para la recepción de la nueva postura antipositivista $^{1}$ y, la instrucción para el ingreso de la Revista de Occidente y de la Biblioteca de Occidente; o al decir de Romero²:

Que aportaron con reiteración y a veces con el carácter de acontecimientos intelectuales, artículos y libros introductores del pensamiento novísimo, pensamiento que por entonces florecía rico en promesas

1 Para Francisco Romero (1952) en Hispanoamérica al darse de manera tardía la crítica y reacción al pensamiento positivista, y el intento de reemplazarla por otras concepciones era algo que debía ocurrir exclusivamente en ambientes universitarios.

2 Se hace referencia a la introducción de la Biblioteca de Occidente de Ortega y Gasset a Latinoamérica. y en realizaciones, anunciando una etapa filosófica de brillo y densidad singulares, y con un extraordinario poder de incitación (Romero,1952, p.45).

Ortega y Gasset no solo fue gestor de la labor de normalizar el pensamiento filosófico de su país, como se sabe atravesó una anormalidad, entendida como el atraso intelectual con respecto a otros países de Europa debido al fenómeno de la "Decadencia Española”; también puso al día a Latinoamérica en el pensamiento occidental de la época mediante la difusión de artículos y de libros introductores, destacándose entre ellos la Revista de Occidente fundada por él en 1923, un medio difusor que representó tanto para España y para Latinoamérica un instrumento normalizador del pensamiento filosófico. Desde los estudios realizados por el colombiano Danilo Cruz se conoce que Ortega y Gasset también hizo presencia en los países de Chile y Uruguay (Cruz, 2014). Con esto, podemos afirmar que el filósofo español contribuyó al proceso de normalización de manera presencial y material.

Para Latinoamérica, Romero es el representante del proceso de la normalidad de la filosofía; su vocación y concientización filosófica lo hacen de cierta manera responsable de esta actividad llevada a cabo en nuestro entorno geográfico. Sin demeritar los estudios y contribuciones que realizó en temas de la cultura, el hombre, la historia de la filosofía, entre otros, se destacó por su gran aporte a la tarea de coordinar la filosofía desde la acade- 
mia, regulada por normas y métodos académicos. Sus categorías "Normalidad Filosófica" y "Fundadores" corresponden a un período en la historia de la filosofía y a una experiencia o forma de hacer esta labor; lo anterior ha sido motivo de discusión que se resume en el estar a favor y en contra de Romero, bajo el indicio de que la Normalidad Filosófica estructuró el quehacer filosófico en nuestra región.

\section{La filosofía en Latinoamérica en el marco de las categorías de Normali- dad Filosófica y Fundadores de Fran- cisco Romero}

La Normalidad Filosófica corresponde al proyecto de Francisco Romero que surgió en Argentina en la primera mitad de siglo XX y posteriormente se extendió a Latinoamérica. En Argentina algunos de los hechos que contribuyeron a la materialización de la Normalidad Filosófica fue la creación de facultades de filosofía en las universidades, la primera, La Facultad de Filosofía y Letras en Buenos Aires en 1896, y por la misma época la Facultad de Humanidades de La Plata; según Romero (1952) otros sucesos que contribuyeron a la normalidad de la filosofía en su país fueron la venida de Ortega y Gasset en 1916 nombrado anteriormente, la fundación del Colegio Novecentista en 1917 y la creación de la Sociedad Kantiana de Buenos Aires.

El término Normalidad Filosófica fue expuesto por Francisco Romero el 15 de septiembre de 1934 en una reunión que el Club de "Poetas, Escritores, Novelistas" conocido con las siglas P.E.N de Buenos Aires organizó al catedrático español Manuel García Morente (1886-1942); en este festejo Romero dirigió el discurso a Morente y dio a conocer que: "lo que faltaba hasta hace poco en España, lo que acaso falta todavía entre nosotros, es lo que llamaré "normalidad filosófica”; quiero decir, la filosofía concebida como común función científica, como trabajo y no como lujo o fiesta" (Romero, 1950, p.130).

Para Romero (1941; 1952) fueron importantes los indicios que se dieron en Latinoamérica por el interés hacia la vocación filosófica; él notó que todos los países de nuestro continente no tenían el mismo grado de curiosidad y capacidad filosófica; observó que la filosofía en algunos lugares era ostentada en unos pocos estudiosos y en otros, la organización del trabajo filosófico era más visible. Lo anterior, lo conllevó a reflexionar que en nuestra región existían grandes dificultades para hacer filosofía, identificando que la mayor de todas era que los países vivían incomunicados, $\mathrm{y}$ en materia de los conocimientos y aportes al pensamiento eran ignorados. A nuestro entender lo anterior corresponde a una de las "condiciones" fundamentales para llegar a la normalidad.

Romero (1941) encontró que en Latinoamérica en el período comprendido entre los años 1939 y 1940 hubo cantidades significativas de libros y artículos de filosofía; él mismo registró que en este lapso de tiempo aparecieron más de cien publicaciones, sin contar aquellas de las cuales aún 
no tenía conocimiento. Para Romero este hecho cuantificable lo consideró como un criterio destacable de la Normalidad Filosófica, y a partir de allí hizo algunos comentarios que terminaron separando, diferenciando y reglamentando el nuevo quehacer filosófico en Latinoamérica; señalando que su época se distinguió por la calidad y el interés notable de la proyección hacia la filosofía que se diferenció de la anterior forma de hacerse porque únicamente "se filosofaba en función de la cátedra, por exigencia de planes de estudio, o el interés personal era tan solitario que constituía rareza y quedaba sin eco efectivo" (Romero, 1941, p.404).

Romero define como Normalidad Filosófica al "ejercicio de la filosofía como una función ordinaria de la cultura, al lado de otras ocupaciones de la inteligencia" (Romero, 1941, p.405). También, como una expresión de la época que manifestó ser "cauce cultural”, que significa, el modo, procedimiento o norma para realizar la filosofía y a la vez para identificar a aquellos que la hacen; asimismo, para Romero (1941) el concepto de Normalidad Filosófica adquiere connotaciones propias de "clima filosófico", "vida filosófica” y "conciencia filosófica”. Podría decirse, que las anteriores ideas explican el proyecto de Romero el cual tiene que ver con la organización de la actividad filosófica, para realizar una verdadera y auténtica filosofía en Latinoamérica.

3 Para Romero (1941) "las expresiones clima filosófico, vida filosófica, conciencia filo-
En este sentido, la filosofía en Argentina y en el resto de Latinoamérica fue entendida desde la perspectiva de actividad "normal"; una etapa o "clima filosófico" donde los países latinoamericanos debían hacer filosofía siguiendo "algunas" prescripciones, señaladas por Romero (1941) como condiciones de la filosofía, tales como: disciplina, seriedad, la información y lectura de escritos filosóficos, y el intercambio mutuo de conocimiento entre los que se ocupan en filosofía, así como también:

Una especie de opinión pública especializada que obra y obrará cada vez más, y según los casos, como estímulo y como represión, como impulso y como freno: esto es, como una vaga, indeterminada sanción continua que antes y después de los juicios expresos de la crítica, corrigiendo lo que hubiera en éstos de partidismo y apreciación individual, promoverá calladamente ciertas cosas, impedirá o dificultará otras, distinguirá planos y establecerá jerarquías (Romero, 1941, p.405).

La etapa de Normalidad Filosófica significó por un lado, que la filosofía debía darse exclusivamente en la universidad porque la institución era la que permitía adquirir la formación seria y disciplinada que necesitaba la filosofía, y de esta manera, no corría el riesgo de ser expuesta en peligro

sófica designan una disposición actual de nuestra cultura y nombran lo que acaso constituye en ella la dimensión más reciente, la novedad más fresca y prometedora” (p.406). 
como se exponía en la formación autodidacta y hecha fuera de las aulas (Romero, 1941). De ahí que una de las tantas condiciones del proyecto de filosofía de Romero era que la filosofía debía "ir más a la escuela" porque en ella "se provee los instrumentos esenciales que garantizan el rendimiento pleno del trabajo individual y practican intercambio de trabajo" (Romero, 1941, p.406).

Por consiguiente, para Latinoamérica entrar en la Normalidad Filosófica y hacer parte de ella suponía contar con condiciones materiales o externas de la filosofía como lo es la Facultad o Departamentos de Filosofía en las universidades, tener cátedras filosóficas, Biblioteca de Filosofía, Editoriales, Revistas de Filosofía; todos los demás medios que posibilitan hacer actividad filosófica académica y ordenar la vida de la filosofía desde la universidad lo que significó estar "en el común cauce cultural” (Romero, 1941, p.404). Además, se puede decir que la Normalidad Filosófica surgió como un fenómeno dado en varias regiones latinoamericanas y corresponde habitualmente con la fecha de creación de la primera institución de Filosofía inaugurada en un país; con lo cual no solo inicia la filosofía de manera normal a la cultura, también se organiza, y sirve de punto de referencia para inaugurar la historia de la filosofía en cada país.

Por otro lado, el propósito de la Normalidad Filosófica de Romero era destacar "la vida y la conciencia filosófica", demostrado en la idea de que cada país en la región latinoamerica- na tenga "la voluntad de agrupación y de mutuo conocimiento entre quienes se consagran a la faena filosófica por profesión o vocación” (Romero, 1941, p.406). Ésta intención materialmente se vio representada con la creación de núcleos o sociedades filosóficas en Latinoamérica que se constituyeron como el signo de la Normalidad Filosófica; entendido también como un requisito y señal que cada país manifestó como muestra del trabajo filosófico sólido y modesto, realizado de manera organizada, coordinada y consciente.

Ahora bien, con respecto a la categoría de los "Fundadores" que surge a la par con el proyecto normalizador de la filosofía, debe decirse que Romero (1952) utilizó este término para referirse a los pensadores que según él fueron merecedores de dar origen a la filosofía en Latinoamérica quienes se caracterizaron por ser adversarios de la doctrina del positivismo. Según Romero (1952), las figuras representativas que iniciaron el proceso de Normalización Filosófica fueron: Alejandro Korn en Argentina, Antonio Caso y José Vasconcelos en México, Alejandro Deustua en Perú, Enrique José Varona en Cuba, Carlos Vaz Ferreira en Uruguay, y Enrique Molina en Chile.

A partir de Romero se entiende que al ingresar la Normalidad Filosófica a cada país de Latinoamérica, surgen en ellos representantes que merecen el nombre de fundadores, de acuerdo a los criterios y "requisitos" que hacen parte de este proceso. Según Romero (1952) era: 
Deber de cada país levantar las figuras que le tocaron en suerte $\mathrm{y}$ pugnar porque sean reconocidas en su aporte y jerarquía, así como es obligación de todos los países hispanoamericanos acoger como propios todos estos varones ${ }^{4}$ eminentes, primera avanzada de una legión que ahora busca conscientemente el mutuo conocimiento, la articulación, la estrecha cooperación futura, convencida de la unidad esencial del destino americano (p.72).

\section{La afirmación y negación de las ca- tegorías de Normalidad Filosófica y Fundadores en Latinoamérica}

La filosofía en Latinoamérica se ha enfrentado constantemente al dilema de su propia definición, de su propia expresión y naturaleza conceptual que desemboca en la polémica sobre la práctica del filosofar desde una perspectiva universalista y localista. Siguiendo a Sasso (1997), las afirmaciones y contradicciones hechas de la normalidad filosófica se sitúan en el plano de definir acerca de "el ser" y "el deber-ser" de la filosofía en nuestro continente, dos tendencias contrarias que se disputan entre lo universal y lo propio de nuestra región, las cuales han ocupado un lugar

4 Es importante decir que la expresión "varones" pertenece también al discurso de la Normalidad Filosófica de Romero, la cual, puede ser entendida como la manifestación y la labor propia de hombres en la producción filosófica realizada en Latinoamérica; al respecto, puede decirse que esta idea o indicio puede interpretase como un canon o criterio para la configuración histórica y epistémica de la filosofía en Latinoamérica. significativo en la producción filosófica de los países latinoamericanos. Por consiguiente, es importante exponer algunas ideas acerca de la Normalidad Filosófica de Romero que se debaten entre la aceptación del proyecto y la negación de éste, con la finalidad de replantearlo para lograr un pensamiento filosófico con características propias de Latinoamérica.

Los filósofos latinoamericanos: el peruano Francisco Miró Quesada (1918-2019) y el mexicano Leopoldo Zea (1912-2004) emprendieron desde el siglo pasado la labor de proyectar el filosofar latinoamericano en la perspectiva histórica del pensamiento occidental, con la finalidad de repensarlo y transformarlo desde las circunstancias latinoamericanas, es decir, un proyecto filosófico desarrollado en Latinoamérica que proviene de la filosofía occidental considerada como auténtica y original.

Tanto para Miró Quesada y Zea, Romero es la figura que representó la conciencia del proyecto filosófico latinoamericano del filosofar; fue el primer latinoamericano que reflexionó sobre la insuficiente e inmadura filosofía en nuestro entorno a causa de la ausencia de posibilidades formativas, y realizó la tarea de comenzar con la actividad filosófica como un proyecto académico. Miró (1974) considera que Francisco Romero fue el "gran forjador" de la filosofía latinoamericana porque contribuyó a superar las dificultades de comunicación que tenían los pensadores de los países latinoamericanos; si se recuerda, para Rome- 
ro el problema de la "incomunicación" antes mencionado era la condición para realizar el proyecto normalizador de la filosofía.

En efecto, el trabajo filosófico de Miró Quesada fue posible gracias a las condiciones académicas adecuadas para una reflexión filosófica:

Las condiciones externas favorables para una producción intensa y continuada, en la que, dejando de lado la visión de la filosofía como propensión, arbitraria y caprichosa, así como el afán de llegar a la creación extraordinaria, se adquiere la conciencia de la obra común (Miró, 1974, p.144).

En este sentido, la historia de la filosofía en Latinoamérica se escribe bajo las condiciones históricas de expresiones históricas de "Normalidad filosófica" y en la que se nombran "Fundadores" de la filosofía; ésta última expresión es clara en el proyecto de Miró Quesada porque continúa con la idea del proyecto de Romero con respecto a la clasificación de los pensadores en la historia de la filosofía latinoamericana. Para reforzar, Miró (1981) los clasifica en tres generaciones: La de los patriarcas, la de los forjadores y la de los técnicos o generación Joven. Los patriarcas corresponden a los antecesores de los forjadores del pensamiento latinoamericano contemporáneo; estos últimos, los forjadores, se caracterizaron por estudiar con seriedad al pensamiento occidental de la época al parecer con tradición filosófica, se diferenciaron de los patriarcas porque éstos care- cieron de una tradición filosófica, en palabras de Miró Quesada una de las características fue la de:

Carecer de una tradición filosófica, y en consecuencia, de la correspondiente formación académica, los patriarcas no logran pasar de un nivel teórico más bien modesto. Esto no significa menor capacidad que la de los discípulos que les sucedieron ni que la de los hombres de la generación técnica (Miró, 1981, p.13).

Para Miró Quesada "los forjadores" o para Romero los "fundadores" fueron los que reconocieron el problema de bajo nivel teórico, llamado por Miró (1981) como "desenfoque teórico" que no dudamos en reconocer como un problema porque este nivel teórico no permite hacer filosofía en sentido académico. Los autores mencionados identifican que dicho asunto se da como consecuencia de la carencia de tradición filosófica por la falta de formación académica. Desde Miró (1981), se reconoce que Romero es el que posibilitó la comprensión para demostrar que la verdadera filosofía en Latinoamérica se da siempre y cuando se tenga una adecuada formación académica, si se tiene un conocimiento de primera mano de los grandes sistemas de la filosofía occidental y se dispone del aparato conceptual necesario para comprenderlo; lo anterior como parte de las condiciones del pensamiento latinoamericano impuestas por la Normalidad Filosófica, creadas con la finalidad de superar el desenfoque teórico, que proviene del quehacer filosófico hecho en condiciones solitarias y de formaciones 
o de-formaciones autodidactas, que para la generación de Romero no conducían a un pensamiento con orientación teórica rigurosa, sistemática y con rasgos característicos.

Otro punto de vista de la Normalidad Filosófica es el propuesto por pensadores de enfoque liberador e intercultural: Horacio Cerutti y Fornet-Betancourt permiten otra mirada de las categorías históricas de la filosofía diferentes a la de Romero. Cerutti (2009) considera que la Normalidad Filosófica y Fundadores de Romero corresponden a una periodización delimitada para la historia de la filosofía latinoamericana que tienen presencia hasta la actualidad; tiene una carga valorativa que ha traído consecuencias como el no tener en cuenta algunos de los pensadores y otras formas de hacer filosofía. Por esta razón, algunos pensadores se han apartado de estas concepciones porque aquella delimitación se ha convirtiendo en un criterio para determinar lo que entra y no en la filosofía académica, entendida "como un verdadero aporte profesional de la filosofía y que debe dejarse fuera. Qué es lo que constituye rigor, pertinencia, fundamentación, calidad filológica, y qué es improvisación, guitarreo, charla de café, manipulación práctica de la filosofía" (Cerutti, 2009, p. 97). En términos de Cerutti (2009):

La normalización lo que hace es establecer un cierto canon. Por una parte, establece quiénes son los que pueden ser considerados fundadores. Por otra, quiénes son los que no merecen ni siquiera el nombre de profesores de filosofía. Quienes son los que merecen ser integrados en la normalización y quiénes son los anormales, los excluidos de la profesión y de la academia (p.94).

Para Cerutti la categoría de Fundadores ha traído consecuencias en la historia del pensamiento filosófico latinoamericano porque limita la presentación de los pensadores, considerando solamente aquellos que reúnen los requisitos para ser reconocidos como los pioneros del quehacer filosófico en nuestro continente. Por otro lado, Fornet-Betancourt (2000) dice que la concepción de Fundadores surgió para indicar a los que han hecho posible el ingreso de la filosofía a Latinoamérica, asegurando con ello que son las figuras representativas e insignes tanto de un contexto local como de toda la región latinoamericana; además, la idea de los fundadores en el marco del concepto de Normalidad Filosófica implica que Latinoamérica sigue la tradición de la filosofía occidental, la cual ingresa como un pensamiento normal, y por consiguiente es el que permite pensar la filosofía como función ordinaria de la cultura. Afirma Fornet-Betancourt (2000):

En la lógica del argumento de Francisco Romero, es legítimo referirse a este grupo de filósofos en el sentido de la tradición fundante de la filosofía en América Latina y darles, en consecuencia, el nombre de "fundadores", pero debe observarse que con esta interpretación Francisco Romero está centrando la importancia de la obra de este grupo de pensadores en el aporte que hace a una determinada cultu- 
ra filosófica, a saber, la cultura filosófica académica que se produce y reproduce con la institucionalización universitaria de la filosofía en Europa (p. 123).

Para Fornet-Betancourt (2000) "la cultura filosófica académica" sería impuesta por la Normalidad Filosófica de Francisco Romero como una forma de hacer filosofía en la cultura latinoamericana, porque ha sido una manera de entender a la filosofía como actividad profesional que se realiza mediante procesos de organización y de institucionalización, imponiéndose como una "cultura dominante" porque establece un orden epistémico y cultural del quehacer filosófico europeo que termina negando, desconociendo y no reconociendo a las "culturas alternativas" presentes en Latinoamérica como "fuente hermenéutica del filosofar”.

La categoría de la generación de los fundadores de Francisco Romero implica que en Latinoamérica se haya optado por seguir la cultura filosófica tradicional de occidente o llamada por Fornet como "cultura filosófica monocultural" que sigue el modelo de la normalidad filosófica de occidente; por tanto, surge como un criterio rector para el desarrollo de la filosofía en nuestro continente porque excluye no sólo a otras formas de expresión filosófica no académicas sino que margina al pensamiento filosófico de las culturas de los pueblos originarios (Fornet-Betancourt, 2000). En este sentido, es importante mencionar el proyecto latinoamericano intercultural del filosofar de Fornet que replantea la tradicional forma de hacer filosofía en nuestra región, donde se propone que para hacer filosofía en sentido plenamente latinoamericano, se debe hacerlo teniendo en cuenta la diversidad y formas de expresión que tiene el pensamiento en las culturas latinoamericanas; a lo que se agregaría también, el compromiso de la academia a filosofar otros contextos sociales.

Por otra parte, dice Cerutti (2009) que Francisco Romero al indicar que la filosofía en la normalidad surge con seres normales y no de genios que tienen disciplina y cumplen ciertas reglas para producir conocimiento filosófico, niega con ello la posibilidad de hacer filosofía de otra manera, y no hay lugar para otras formas de expresión o filosofares; por consiguiente, la filosofía hecha en la escuela y para la escuela, es decir la académica, queda limitada y encerrada a un círculo elitista que reflexionan problemas académicos.

Además, Cerutti (2009) considera que para Francisco Romero era más importante la historia de la filosofía que la historia de las ideas, porque la primera se enfoca a un saber riguroso y normalizado alejado de contextos sociales, mientras que el propósito de la segunda es la reconstrucción de cosmovisiones, las cuales al no ser tan rigurosas y claras como la filosofía se convierten en un trabajo secundario. No obstante, afirma Miró (1974) que Romero estableció que la historia de la filosofía era el primer elemento en el que debía formarse todo filósofo latinoamericano; y por consiguiente, 
fue esta formación la que contribuyó a que las nuevas generaciones adquieran el interés para desarrollar la historia de las ideas. Es decir en nuestra opinión, la historia de la filosofía se convierte en el fundamento de la historia de las ideas; en otras palabras la historia de la filosofía occidental sería una especie de instrumento para pensar a Latinoamérica.

\section{Las categorías de Normalidad Filosó- fica y Fundadores en el contexto co- lombiano}

La fundación del Instituto de Filosofía en el año 1945 adscrito a la Escuela de Derecho de la Universidad Nacional representa un hito importante en la historia de la filosofía en Colombia. El Instituto de Filosofía desde la perspectiva de Francisco Romero significaría la normalización de la filosofía porque ya es considerada como una actividad normal, aceptada en la cultura colombiana. Con la inauguración del Instituto de Filosofía, también se reconoce a los representantes y fundadores de la filosofía, quienes hicieron posible la institucionalización de la filosofía: Rafael Carri$\mathrm{llo}^{5}$, Cayetano Betancur, Luis Eduardo Nieto Arteta, Abel Naranjo Villegas, y Danilo Cruz.

En el proceso de normalización, es decir, de profesionalización e institucionalización de la filosofía señalamos como elementos fundamentales:

5 En la entrevista realizada al filósofo Rafael Carrillo (1907-1996) el primer director del Instituto de Filosofía, por el colombi-
1. La universidad. EL Instituto de Filosofía de la Universidad Nacional, la institución para dar a conocer a la filosofía como una disciplina de estudios e investigación; y en los años 60-80 como profesión gracias a la creación de diversas Facultades, Departamentos y Centros de Estudio de Filosofía en todo el país.

2. La creación de las revistas especializadas en filosofía. La primera en aparecer fue la Revista Colombiana de Filosofía en el año 1948, creada por los profesores Adalberto Botero Escobar y Abel Naranjo Villegas.

3. La conformación de las comunidades o sociedades filosóficas. La primera en el país fue La Sociedad Colombiana de Filosofía -SCFcreada desde el año 1957, vigente hasta la actualidad (año 2020).

4. La creación de eventos periódicos como Foros y Congresos de Filosofía. El primer Foro Nacional de Filosofía se realizó en la Universidad de Nariño ciudad de Pasto

ano Numas Gil encontrada en Reportaje a la filosofía Tomo I (1993), menciona que un hecho decisivo para la fundación del instituto fue la carta que algunas figuras destacadas de la academia como el profesor López de Mesa, el doctor Gerardo Molina y Germán Arciniegas enviaron a las directivas de la Universidad Nacional solicitando la creación del Instituto. Una vez fundado el Instituto bajo la dirección de Rafael Carrillo hicieron parte de éste los colaboradores más cercanos del director: Danilo Cruz, Jaime Jaramillo Uribe, Jaime Vélez Sáenz, Cayetano Betancourt, López de Mesa, Luis Eduardo Nieto Arteta. 
en el mes de junio del año $1975^{6}$. El primer congreso de filosofía latinoamericana fue realizado en la ciudad de Bogotá en el año 1980 por la Universidad Santo Tomás ${ }^{7}$.

6 La Universidad de Nariño contaba en su estructura académica con un Departamento académico exclusivamente dedicado a la filosofía. En este Departamento se gestó liderado por los docentes (Rosa Cristina Martínez Rodríguez, José Miguel Wilches Castrillón, Alfredo Rodríguez Pantoja, Dumer Mamian Guzmán, Miguel Angel Ochoa Barón, Pedro Pablo Rivas Osorio, Raúl Gómez Quintero -Director del Departamento- y los estudiantes Héctor Rodríguez Rosales y Álvaro Castro López) el Primer Foro Nacional de Filosofía. En la actualidad se han realizado Foros en diversas universidades de Colombia lo que le ha dado la característica de ser itinerante. Uno de los resultados de este Foro fue el Foro de Estudiantes de Filosofía del cual en la actualidad también se han realizado en diversas universidades de Colombia. Es de recordar que el docente Pedro Pablo Rivas Osorio presentó como propuesta llamarlo Ágora por ser ésta un punto de encuentro de discusión y no Foro por las fuertes connotaciones restrictivas del uso del término. Se decidió denominarlo Foro y darle la apertura a todas las tendencias y corrientes filosóficas que en el momento se daban en el país. Así nació el Primer Foro Nacional de Filosofía realizado el 28, 29,30 de junio de 1975 liderado por el Departamento de Filosofía de la Universidad de Nariño en la ciudad de Pasto, Colombia.

7 El I Congreso Internacional de Filosofía Latinoamericana estuvo dedicado al estudio de las problemáticas de la filosofía latinoamericana: Historia de la filosofía latinoamericana, tomismo y metafísica en América Latina y filosofía política. Según las recopilaciones hechas por Germán Marquínez Argote en el libro La filosofía en Colombia-Historia de las ideas (1992) a este congreso asistieron más de cuatrocientos congresistas; algunos pensadores internacionales: Leopoldo Zea, Enrique
Para Juan Camilo Betancur "desde la historia de las ideas filosóficas no se habla de una "etapa de normalidad filosófica" sino de una "normalización filosófica”” (Betancur, 2015, p.147). Como se puede notar, la expresión con Francisco Romero era de Normalidad Filosófica, pero en el contexto colombiano tiene otra connotación: un proceso inacabado de la filosofía; esto quiere decir que la filosofía en Colombia para la época no había alcanzado su desarrollo y madurez plena.

Algunos autores colombianos que han estudiado y discutido el tema de la normalización filosófica son Leonardo Tovar, Damián Pachón Soto, Carlos Arturo López y Juan Camilo Betancur. En ellos se identifica una discusión en torno a la idea de periodización, definición, clasificación de los fundadores de la filosofía y el no reconocimiento de algunos filósofos en la historia de la filosofía como: Carlos Arturo Torres, Julio Enrique Blanco, Fernando González, Rafael Gutiérrez Girardot, Víctor Florían, Jorge Aurelio Díaz, Nicolás Gómez Dávila, Estanislao Zuleta, Darío Botero Uribe, Santiago Castro-Gómez (López, 2018); (Pachón, 2017, 2011); (Rodríguez, 2003); (Tovar, 1998).

Dussel, Hugo Assmann, Juan Carlos Scannone, Mario Sambarino, Hilton Iappiassú, Luisa Rivara, entre otros; filósofos colombianos: Guillermo y Jaime Hoyos, Julio César Arroyave, Magdalena Holguín, Rafael Torrado, Francisco Sierra, Eugenio Lákatos, Jairo Muñoz, Jaime Rubio, Germán Marquínez, Roberto Salazar, Luis José González, etc. (Marquínez, 1992). 
Con respecto a la periodización, se considera que la normalización filosófica en nuestro país no tiene únicamente como punto de referencia la fundación del Instituto de Filosofía. Según Tovar (1998), el estudio, lectura y producción de la filosofía moderna basada en pensadores alemanes se daría en los años 20 del siglo XX mucho antes de la fundación del instituto:

No debemos asumir simplemente que sólo desde 1964 se conocieron en Colombia los nombres de Cassier, Husserl, Scheler, Bergson y Heidegger. Una somera revisión de las publicaciones de la época indica que desde mucho antes había personas con interés particular por el filosofar, no en pocas ocasiones con un notable grado de especialización, y quizá con mayor voluntad creativa de la estilada después. Basta mencionar al barranquillero Julio Enrique Blanco (1890-1985), quien desde la segunda década del siglo dio a la luz estudios en torno a filósofos modernos y contemporáneos (Tovar, 1998, p.20).

Desde otra perspectiva, para Pachón (2011) la normalización filosófica en Colombia es posible a partir de los años setenta del siglo XX, como consecuencia de la formación y fruto de las enseñanzas que recibieron los primeros discípulos de los maestros que viajaron a estudiar a Alemania y regresaron en los años sesenta. Como se sabe, ciertos profesores del Instituto de Filosofía que merecen recordarse como Danilo Cruz Vélez y Rafael Carrillo, entre otros, estudiaron filosofía en Alemania y los aportes de sus formaciones en Colombia serían lo que confirmaría el inicio de la normalización. Pero, Pachón (2011) considera que para hablar de la normalización filosófica en Colombia es necesario tener en cuenta el desarrollo de la filosofía y los intelectuales de los últimos treinta años del siglo XX y lo que corresponde al siglo XXI como parte del proceso de la normalización de nuestro país. Por mencionar algunos maestros significativos que contribuyeron al fortalecimiento y a la formación de la filosofía colombiana: Rafael Gutiérrez Girardot, Daniel Herrera Restrepo, Guillermo Hoyos Vásquez, Rubén Sierra Mejía, entre otros.

Es importante decir que la etapa de la normalización de la filosofía corresponde a un proceso de la filosofía en la historia colombiana; entonces, puede identificarse que existe un momento previo y uno posterior de la normalización filosófica, los cuales podrían denominarse como un momento pre-normalizador y post-normalizador de la filosofía, separados por la fase de periodización y forma de hacer filosofía en Colombia que está influenciada por las condiciones y características del proyecto de normalidad filosófica de Romero.

Con respecto a algunos de los aportes hechos al concepto de normalización filosófica en Colombia, se puede destacar a Danilo Cruz Vélez (19202008) quien describe a esta etapa de la filosofía como una "anormalidad filosófica”, para señalar que el rasgo distintivo del pasado filosófico de nuestro medio es haber tenido una filosofía “anómala” (Cruz, 2014). Igual- 
mente, para Guillermo Hoyos (19352013) esta fase corresponde a una "normalidad tardía” (Hoyos, 1999). Y para Rubén Sierra la filosofía que se dio en los años 40 del siglo XX pertenece a un "cambio de actitud" porque representa la línea de ruptura de la forma tradicional de hacer filosofía; afirma Sierra (1985):

Un cambio de actitud, pues ahora se entiende que la filosofía es un campo de saber que requiere del estudio de su historia, del dominio de sus categorías o conceptos, de un manejo de su metodología o metodologías, y sobre todo que es una disciplina a la que hay que llegar desprovisto del temor a perder la fe (p.10).

Para Sierra (1985), la normalización de la filosofía o la producción filosófica realizada de manera institucionalizada ha traído como consecuencias el marginamiento al trabajo filosófico colombiano desde otras expresiones, como también la marginación desde la academia, observada por ejemplo en la carencia de fuentes de trabajo intelectual distintas a las que se estudia o se reglamenta en las cátedras, la ausencia de medios institucionales como editoriales, institutos de investigación etc., que impiden el desarrollo pleno de la filosofía en Colombia.

Siguiendo a Sierra (1982), la normalización filosófica dada en nuestro país no es una actividad para determinar que la filosofía en Colombia cumplió "mayoría de edad", porque surgió como un ejercicio improvisado y una profesión limitada a las circuns- tancias universitarias; es decir, que la profesionalización de la filosofía tiene condiciones normativas impuestas por la academia que limitan al quehacer filosófico ya sea por preferencia de autores, conceptos, corrientes, textos, entre otros. Por otra parte, para Cruz (2014):

La institucionalización encierra en sí el peligro de la burocratización, que es muy negativa, porque la mayoría de los profesores de filosofía tienden, por lógica interna de las cosas, a convertirse predominantemente en funcionarios, cuya suprema aspiración no es el saber por el saber, inherente a la filosofía, sino más bien escalar un grado superior en el escalafón académico (p.133).

En este sentido, el ambiente institucional burocratiza la actividad filosófica porque se limita únicamente a las condiciones académicas, reconociendo el quehacer filosófico realizado desde estas instancias, marginando otras formas de hacer y producir la filosofía.

La idea de marginación y exclusión de pensadores en la historia de la filosofía colombiana es una cuestión que resulta de la normalización filosófica, enmarcada desde las circunstancias normativas que impulsó el proyecto de Francisco Romero en Latinoamérica; en el caso colombiano no ha sido una excepción referirse al tema de normalización filosófica como una posición para narrar la historia de la filosofía, partiendo de la clasificación $\mathrm{y}$ reconocimiento de los fundadores o 
Rivas Osorio, P. P., Deraso Andrade, M. A. (2020). La categoría de normalidad filosófica de Francisco Romero reguladora de la historia de la filosofía en Latinoamérica y Colombia. Estudios Latinoamericanos, 46-47, 53-71.

pioneros de la filosofía en este contexto. Al respecto, Carlos Arturo López afirma:

Durante varias décadas, quizás demasiadas, la historia de la filosofía en Colombia fue contada por los filósofos profesionales. Proyectando sobre esa historia su propio entendimiento del oficio, afirmaban que la filosofía comenzó en el momento de su profesionalización. Antes de ese momento, vale decir, antes del surgimiento de la filosofía como disciplina autónoma, de la aparición de revistas especializadas, de la formación de profesores familiarizados con el universo conceptual de los autores clásicos, estudiados en sus lenguas originales, no existía propiamente la filosofía en Colombia. Lo que había era tal vez "pensadores", ensayistas, intelectuales diletantes o "ideólogos" vinculados a los partidos políticos, pero no filósofos en el sentido propio de la palabra. Tal es el consabido relato de la "normalización" de la filosofía" (López, 2018, p.11).

Para Carlos Arturo López el relato normalizador es "un criterio ahistórico para toda forma de hacer filosofía, los normalizadores exigen que los textos escritos antes de 1930 cumplan con los requisitos formales definidos por ellos, recriminándoles así el carácter prefilosófico" (López, 2018, p.14). La filosofía en Colombia tuvo reconocimiento de manera institucional y a partir de allí se configuró su historia, descalificando el quehacer filosófico de otros pensadores tenidos como "autodidactas", "aficionados", "no sistemáticos" "faltos de rigurosidad conceptual".
Luego:

La historia de la filosofía de los filósofos apoyada en el marco de referencia de la modernidad construye un pasado filosófico nacional para legitimar las "nuevas" formas de hacer filosofía que la generación de los filósofos de la normalización promueve. Esto permite mostrar también cómo la historia de la filosofía suscribe los valores con que los normalizadores definieron el oficio del filósofo; valores atados a unas condiciones históricas que marcaron las pautas del oficio en el presente (López, 2018, p.285).

Guillermo Rodríguez, Carlos Arturo López y Juan Camilo Betancur han propuesto reflexionar la historia de la filosofía en Colombia desde otras posibilidades. En el caso de Rodríguez (2003) propone que la historia de la filosofía en Colombia debe hacerse en relación con la vida y la sociedad, $\mathrm{y}$ fortalecerse desde los pensadores quienes han realizado pensamiento en condiciones de exilio como una muestra de hacer pensamiento alejado de los entornos académicos. Son importantes para la historia de la filosofía quienes han hecho y hacen filosofía de la acción encaminada a reflexionar las circunstancias y problemas de nuestra realidad.

Por otra parte, López (2018) considera que la escritura filosófica exigida desde la academia como parte de la normalización filosófica colombiana, es excluyente de la producción filosófica de autores de obras contemporáneas al episodio normalizador, por tanto, plantea una historia de la 
filosofía en Colombia que se narre desde las condiciones de posibilidad de la escritura filosófica:

Este trabajo trata entonces de la historia de la escritura, en tanto esta se presenta como un hacer regular regido por condiciones históricas relativas a la escritura misma. Un hacer atado a los vestigios materiales en los que se concreta esa práctica: un conjunto indefinido de textos. Por ello, hará falta volver sobre los libros y las publicaciones seriadas que en su tiempo fueron reconocidos como filosofía, a las publicaciones que se le acercaron haciendo comentarios de textos filosóficos nacionales o foráneos, a las que usaron las referencias de su tradición intelectual y a las que ofrecieron una interpretación de ella (López, 2018, p.17).

Por otro lado, Betancur (2015) propone una reescritura de la normalización filosófica a partir de las experiencias y significados que ha tenido el concepto de normalización en la historia de la filosofía colombiana:

Finalmente, una rescritura de la historia de la "normalización filosófica” será posible mostrando la multiplicidad de significados que ha tomado el concepto de "normalización" en las historias escritas, así como el desfase entre los significados que han ido predominando en ellas y las pretensiones iniciales que venían expresadas bajo la idea de filosofía o la de normalización (Betancur, 2015, p.153).

De lo expuesto podríamos tener algunas conclusiones, a más de las que otros investigadores y estudiosos del tema podrían aportarnos: la periodización del quehacer filosófico en un antes y un después determinados por la categoría de Normalidad Filosófica de Romero que representa un proyecto de filosofía realizado de manera local, que gracias a su importancia no sólo contribuyó al desarrollo de la filosofía y a la de su historia en la Argentina, sino que terminó trascendiendo y proyectándose en Latinoamérica.

El discurso de la Normalidad Filosófica y Fundadores de Romero es una interpretación para la historia de la filosofía en Latinoamérica que queda expuesto a dos grandes perspectivas; la primera, propicia la forma de reconocer y acercarnos a las teorías y problemas tradicionales de la filosofía; y la segunda, a otros modos de ejercer la actividad filosófica partiendo de la situación social, económica y política de los países y las sociedades, por tanto, ambas aportan a la historia de las ideas filosóficas en América latina; quizá esta última mirada posibilita más alternativas para explorar y bosquejar un pensamiento procedente de realidades diversas como proyectos de pensamiento latinoamericano.

Para la historia de la filosofía en Colombia la categoría de Normalidad tuvo como consecuencia su periodización y una experiencia de hacer filosofía; un modelo de filosofía caracterizado por darse exclusivamente desde la academia, desarrollado con métodos validados como rigurosos por cultivadores de determinadas corrientes filosóficas: la academia determina el 
canon de lo que es y no es filosofía; como consecuencia la exclusión de otras formas del quehacer filosófico que pueden tenerse como fundamentales en la historia de la filosofía y en la historia de las ideas filosóficas. De aquí una conclusión más: en Colombia hay una relación entre el desarrollo de la Universidad colombiana y la academia, la política y la filosofía que propicia temas y espacios intelectuales para ser investigados. 


\section{Referencias}

Betancur, J. (2015). Para un análisis crítico del concepto de normalización filosófica. Universitas Philosophica, 32 (65), 137-158.

Cerutti, H. (2009). "Normalización” y después. En. H. Cerutti (Ed), Seguimos filosofando. (pp. 89-105). La Habana, Cuba: Ciencias Sociales. Recuperado de https://pt.scribd.com/document/399724525/Cerutti-Y-seguimos-filosofando-pdf [consultado el 30 marzo 2020].

Cruz, D. (2014). Tabula rasa volumen IV obras completas. Bogotá, Colombia: Ediciones Universidad de los Andes, Universidad de Caldas, y Universidad Nacional de Colombia.

Dussel, E. (1970). Francisco Romero, filósofo de la modernidad en la Argentina. Cuyo Anuario de Historia del Pensamiento Argentino, VI, 79-106. Recuperado de https://bdigital.uncu.edu.ar/app/navegador/?idobjeto $=4349$ [consultado el 25 mayo 2020]

Fornet-Betancourt, R. (2000). Para un balance crítico de la filosofía latinoamericana en la llamada etapa de los fundadores. Cuyo Anuario de filosofía Argentina y Americana, 17, 117-132. Recuperado de https://bdigital.uncu.edu.ar/app/ navegador/?idobjeto $=1018$ [consultado el 20 enero 2020]

Gil, N. (1993). Reportaje a la filosofía tomo I. Bogotá, Colombia: Punto Inicial.

Hoyos, G. (1999). Medio siglo de filosofía moderna en Colombia. Reflexiones de un participante. Revista de estudios sociales, (3), 43-58.

López, C. (2018). El terreno común de la escritura: una historia de la producción filosófica en Colombia, 1892-1910. Bogotá, Colombia: Pontificia Universidad Javeriana.

Marquínez, G. (1992). La filosofía latinoamericana. En A. Marquínez et al, (Eds.), La filosofía en Colombia-Historia de las ideas (pp.423-451). Bogotá, Colombia: El Búho.

Miró, F. (1981). Proyecto y realización del filosofar latinoamericano. México D.F., México: Fondo de cultura económica.

Miró, F. (1974). Despertar y proyecto del filosofar latinoamericano. México D.F., México: Fondo de Cultura Económica.

Pachón, D. (2011). Estudios sobre el pensamiento colombiano volumen I. Bogotá, Colombia: Ediciones desde abajo.

Pachón, D. (2017). Cien años de filosofía en Colombia (1910-2010). En torno a la lectura de Juan José Botero. Ideas y Valores, LXVI, (164), 413-421. 
Rodríguez, M. (2003). La filosofía en Colombia: modernidad y conflicto. Rosario, Argentina: Laborde Editor.

Romero, F. (1952). Sobre la filosofía en América. Buenos Aires, Argentina: Raigal.

Romero, F. (1950). El hombre y la cultura. Buenos Aires, Argentina: Espasa-Calpe.

Romero, F. (1941). Sobre la filosofía en Iberoamérica. Universidad Católica Bolivariana, 6 (19-20), 403-409.

Sasso, J. (1997). La filosofía latinoamericana y las construcciones de su historia. Caracas, Venezuela: Monte de Ávila Editores Latinoamericana.

Sierra, R. (1985). La filosofía en Colombia siglo XX. Bogotá, Colombia: Procultura.

Sierra, R. (1982). Temas y corrientes de la filosofía colombiana en el siglo XX. Cuadernos de filosofía latinoamericana, 12,78-101.

Tovar, L. (1998). La normalización de la filosofía en Colombia. Cuadernos de filosofía latinoamericana, (72-73), 19-25. 